\title{
Reduced Subjective Response to Acute Ethanol Administration Among Young Men with a Broad Bipolar Phenotype
}

\author{
Sarah W Yip', Joanne Doherty ${ }^{2}$, Judi Wakeley', Kate Saunders', Charidimos Tzagarakis', Harriet de Wit ${ }^{3}$, \\ Guy M Goodwin' and Robert D Rogers*,' \\ 'Department of Psychiatry, University of Oxford, Oxford, UK; ${ }^{2}$ Institute of Psychological Medicine and Clinical Neurosciences, Cardiff University, \\ Cardiff, UK; ${ }^{3}$ Department of Psychiatry and Behavioral Neuroscience, University of Chicago, Chicago, IL, USA
}

\begin{abstract}
Elevated lifetime prevalence rates of alcohol use disorders (AUDs) are a feature of bipolar disorder (BD). Individuals at-risk for AUDs exhibit blunted subjective responses to alcohol (low levels of response), which may represent a biomarker for AUDs. Thus, individuals atrisk for $\mathrm{BD}$ may exhibit low responses to alcohol. Participants were 20 unmedicated adult males who reported high rates of hypomanic experiences (bipolar phenotype participants; BPPs), aged 18 to 21 years, and 20 healthy controls matched on age, gender, IQ, BMI, and weekly alcohol intake. Subjective and pharmacokinetic responses to acute alcohol $(0.8 \mathrm{~g} / \mathrm{kg})$ vs placebo administration were collected in a randomized, double-blind, cross-over, placebo-controlled, within-subjects design. BPP participants reported significantly lower subjective intoxication effects ('feel high': $F=14.2, p=0.001$; 'feel effects': $F=8.1, p=0.008$ ) across time, but did not differ in their pharmacokinetic, stimulant, or sedative responses. Paradoxically, however, the BPP participants reported significantly higher expectations of the positive effects of alcohol than controls. Our results suggest that unmedicated young males with previous hypomanic experiences exhibit diminished subjective responses to alcohol. These blunted alcohol responses are not attributable to differences in weekly alcohol intake, pharmacokinetic effects (eg, absorption rates), or familial risk of AUDs. These observations suggest that the dampened intoxication may contribute to the increased rates of alcohol misuse in young people at-risk for BD, and suggest possible shared etiological factors in the development of AUDs and BD.
\end{abstract}

Neuropsychopharmacology (2012) 37, 1808-18I5; doi:I0.1038/npp.2012.45; published online II April 2012

Keywords: alcohol abuse/dependence; bipolar disorder; adolescence; hypomania; mood; ethanol

\section{INTRODUCTION}

Bipolar disorder (BD) is one of the leading causes of disability worldwide (World Health Organization, 2008), and is increasingly recognized as a continuum of symptomatology, ranging from experiences of mild hypomania to extreme bouts of elevated mood (mania) and depression. Traditionally, research studies have focused on the more severe end of the bipolar spectrum. Although studies in this population have indisputably helped to inform current understanding of the BDs, they are nonetheless limited by the confounding factors inherent in studying a chronically ill population; for example, the effects of long-term medication, multiple hospitalizations, comorbidities, cognitive

*Correspondence: Professor RD Rogers, Department of Psychiatry, University of Oxford, Warneford Hospital, Oxford, Oxfordshire OX3 7JX, UK, Tel: + 4401865 613106, Fax: + 440 1865 793101,

E-mail: robert.rogers@psych.ox.ac.uk

Received 8 November 2011; revised 8 March 2012; accepted 12 March 2012 impairment, and long disease histories. Recent research has sought to eliminate these confounds by studying at-risk individuals for $\mathrm{BD}$, identified on the basis of previous hypomanic experiences (Chandler et al, 2008; Calabrese et al, 2003). Such individuals - hereafter referred to as individuals with the bipolar phenotype (BPP) - are at increased risk for a range of mental health problems including, but not limited to, BD (eg, anxiety, depression; Chandler et al, 2008; Calabrese et al, 2003). Moreover, among individuals with the BPP some already meet DSM-IV-TR criteria for bipolar II or NOS (Rock et al, 2010; Chandler et al, 2009) and their symptoms and alterations in emotional processing are relatively homogeneous. Follow-up of young people with the BPP indicates that intermittent mood elevation in young people is a significant predictor of a variety of subsequent mental health problems even in the short term (Tijssen et al, 2010).

Public health policy increasingly recognizes the importance of identifying biomarkers for vulnerability to mental health disorders (eg, National Institute of Mental Health). Focusing on at-risk populations for BD affords significant 
methodological advantages. First, as described above, this approach avoids the confounding effects of the consequences or epiphenomena of chronic illness. Second, bipolarity is now recognized to represent a spectrum involving a much larger proportion of mood disorder populations than just the paradigm illness, bipolar I disorder (Merikangas et al, 2007). Therefore, its manifestations across a broader spectrum may contribute to societal problems associated with alcohol and substance misuse, other addictions and impulse control disorders.

Epidemiological studies indicate that lifetime alcohol use disorders (AUDs) are significantly more prevalent among individuals with BDs (46-61\% in BD I; $36-39 \%$ in BD II), exceeding the rates reported in schizophrenia and major depression (Regier et al, 1990; Kessler et al, 1997; Merikangas et al, 2007). Clinically, individuals with both AUDs and BD have higher rates of experienced physical or verbal abuse (Frye et al, 2003) and suicide attempts (Frye et al, 2003), poorer treatment compliance (Strakowski et al, 1998), and higher relapse rates (Tohen et al, 1990) compared with BD patients alone. However, the etiology of AUDs in BD has not been previously explored experimentally (Le Strat and Gorwood, 2008). The high prevalence of AUDs (49.3\% in a voluntary registry of BD I patients; Chengappa et al, 2000) means that studying the acute effects of alcohol in patients with mature $\mathrm{BD}$ might be confounded by previous alcohol use.

Individuals at-risk for AUDs typically report diminished subjective experiences or 'low-level responses' (LLRs) to alcohol administration (Trim et al, 2009; Schuckit and Smith, 2011) although this has not been found across all studies (eg, King et al, 2002). LLRs at ages 19 and 20 predict AUDs at 10- and 8-year follow-up, respectively (Volavka et al, 1996; Schuckit and Smith, 1996). In contrast, other studies have reported that individuals at-risk for AUDs (Conrod et al, 1997; Peterson et al, 1996) exhibit enhanced physiological responses to alcohol (eg, elevated heart rate; elevated plasma $\beta$-endorphins), consistent with the so-called 'differentiator' model. The 'differentiator' model proposes that individuals at-risk for AUDs experience enhanced stimulant effects during the ascending limb of the blood alcohol curve and diminished sedative effects during the descending limb (reviewed in Ray et al, 2010; Newlin and Thompson, 1990; Newlin and Renton, 2010). Although there remains some uncertainty about which precise dimension of individuals' subjective reactions to alcohol is most predictive of subsequent AUDs (King et al, 2002, 2011), there is consensus that some aspect of individual subjective response mediates the risk for-and development of-AUDs, making such response profiles potentially interesting endophenotypes (Morean and Corbin, 2010; Ray et al, 2010).

Here, we tested the hypothesis that BPP individuals show a dampened subjective response to alcohol. As proposed by Schuckit in his LLR theory, people with the BPP might drink more in order to experience alcohol's normative effects. However, given the conflicting findings of the literature, it is also possible that people with the BPP show greater stimulant effects during the ascending limb. We included only males in our experiment because problem drinking is more prevalent in men, and LLRs have been most robustly observed among men at-risk for AUDs (Quinn and Fromme, 2011).
Finally, alcohol consumption is also influenced by beliefs about its cognitive, emotional, and behavioral effects (Brown, 1985; Christiansen et al, 1989), and expectations are known to influence both responses to acute alcohol and the amount of alcohol consumed (Brown et al, 1987). Alcohol-related expectancies may be especially prominent during the adolescent years, contributing to subsequent alcohol misuse and dependence (Brown, 1985). Therefore, we also asked our participants to complete the Alcohol Expectancy Questionnaire (AEQ; Brown et al, 1987) to identify whether individuals with the BPP have heightened expectancies about the positive consequences of alcohol consumption.

\section{PARTICIPANTS AND METHODS}

\section{Participants and Recruitment}

The study protocols were approved by Oxford University's Central University Research Ethics Committee. Forty medication-free male participants between the ages of 18 and 21 were recruited via advertisements or from an on-line survey (Chandler et al, 2008). Participants were selected according to their scores on the Mood Disorders Questionnaire (MDQ; Hirschfeld et al, 2000), a self-report instrument for screening $\mathrm{BD}$ (specificity=0.90). The MDQ includes $13 \mathrm{yes} / \mathrm{no}$ questions, based on clinical observations and the DSM-IV diagnostic criteria for BDs (eg, 'Has there ever been a period of time when you were not your usual self and thoughts raced through your head or you couldn't slow your mind down?'; Hirschfeld et al, 2000). Originally designed and validated for use in outpatient populations, the MDQ has since been validated in the general population (specificity $=$ 0.97; Hirschfeld et al, 2003), and has been used to define the common bipolar phenotype in terms of elevated mood in young people (Rock et al, 2010). Specifically, two participant groups were recruited: participants with MDQ scores $=0$ (control participants), and participants with MDQ scores $\geqslant 7$ (BPP). After the study was described to the subjects, written informed consent was obtained.

Participants were assessed using the Mini International Neuropsychiatric Interview (Sheehan et al, 1998) and screened to exclude any major psychiatric illness - including alcohol and substance abuse/dependence-with the exception of BD I, II or NOS for the BPP participants. Other alcohol-related exclusion criteria included (i) a first-degree relative with a history of an AUD; (ii) any psychotropic medication; (iii) any non-psychotropic medication that might interact with alcohol; (iv) significant physical health problems for which drinking alcohol would be inadvisable; (v) an absence of previous exposure to alcohol; (vi) any history of alcohol-related problems (eg, seizures, blackouts); and (vii) daily alcohol consumption in excess of the advised healthy levels of consumption recommended by the UK government (ie, 3-4 units per day for males; www.dh.gov.uk), as assessed by the Alcohol Use Questionnaire (Mehrabian and Russell, 1978).

Participants also completed psychometric assessments of depressive symptoms (Quick Inventory for Depressive Symptomatology; QIDS; Rush et al, 2003), impulsivity (Barratt Impulsiveness Scale; BIS-11; Patton et al, 1995), neuroticism (Eysenck Personality Questionnaire's Neuroticism subscale; EPQ-N; Eysenck et al, 1985), intelligence 
(Raven et al, 1998), and positive alcohol expectancies (AEQ; Brown et al, 1987). The AEQ is a true-false questionnaire composed of statements about the effects of alcohol (eg, 'Alcohol can transform my personality') that correspond to six expectancy subscales-global positive changes, sexual enhancement, social and physical pleasure, social assertiveness, relaxation, arousal/aggression - the reliability and internal consistency of which have been demonstrated previously (Goldman et al, 1997).

\section{Study Procedures}

Participants attended two experimental study visits at the University of Oxford's Department of Psychiatry at the Warneford Hospital, scheduled at least 1 week apart. Each study visit began at 13:30 hours. Participants were randomized using a fixed, double-blind, cross-over, withinsubjects design to receive either active $(0.8 \mathrm{~g} / \mathrm{kg}$ ethanol) or placebo doses at study visits one or two. Participants were required to abstain from alcohol use for at least $24 \mathrm{~h}$ and from caffeine use for at least one hour before each visit. All participants consumed a standardized light lunch one and a half hours before each visit. At the start of each study visit, participants completed baseline measurements of pulse, blood pressure, breath alcohol concentration $(\mathrm{BrAC})$ and subjective alcohol effects (see below).

Following baseline measures, participants consumed one of two beverages (ie, placebo or active dose). Both beverages consisted of $90 \%$ ethanol, diet tonic water, and concentrated lime juice. Active beverages contained $0.8 \mathrm{~g} / \mathrm{kg}$ ethanol (active dose). Placebo beverages contained a $1 \%$ ethanol mask. The total volume of each beverage was $450 \mathrm{ml}$ for a $70 \mathrm{~kg}$ participant, with adjustments made for weight. Beverages were consumed according to a cumulative dosing schedule, with the total beverage divided into nine $50 \mathrm{ml}$ portions, and portions consumed at 3-min intervals. In order to create an additional taste and odor mask, the rim of the cup used to administer the beverages was dipped in gin before each dose (for both active and placebo beverages). Following consumption of the beverages, participants completed measurements of subjective ratings at the 30 , $45,60,120$, and 180 -min time-points. Pulse readings were also taken at the $30,60,120$, and 180-min time-points. BrAC was measured at the 60,120 , and 180 -min time-points. In order to ensure that the primary researcher remained blind, $\mathrm{BrAC}$ and pulse ratings were taken by another researcher.

\section{Measures of Subjective Effects}

The subjective effects of acute ethanol administration were measured in two ways: (i) using a visual analog rating-scale of drug effects (the Drug Effects Questionnaire; DEQ; Kirk and de Wit, 2000) and (ii) using the Biphasic Alcohol Effects Scale (BAES; Martin et al, 1993). The DEQ consists of four visual analog scales linked to the following questions: 'Do you feel any drug effects?', 'Do you like the effects you are feeling right now?', 'Are you high?' and 'Would you like more of what you consumed, right now?' Participants rated their responses along a $100 \mathrm{~mm}$ line ranging from 'none/not at all' $(0 \mathrm{~mm})$ to 'a lot/very much'. The sensitivity of the DEQ across different psychoactive substances, including alcohol, has been demonstrated previously (Kirk and de Wit, 2000; White et al, 2005). The BAES is a 14-item adjective rating scale of alcohol's stimulant and sedative effects, with scale items ranging from 0 ('not at all') to 10 ('extremely'). Other studies report internal consistency scores of 0.94 and 0.95 for the stimulation subscale, and of 0.91 and 0.93 for the sedation subscale, during the ascending and descending limbs of intoxication, respectively (Rueger et al, 2009).

\section{Statistical Analyses}

Between-group comparisons of demographic, psychometric, and alcohol-related variables were completed using univariate analyses of variance (ANOVAs). BrAC, pulse, and blood pressure were compared using repeated-measures ANOVAs with the between-subjects factors of group and order and the single within-subject factor of time. Analyses of DEQ and BAES subscale data were conducted in several steps. First, between-group differences in placebo scores were examined using repeated-measures analyses with the between-subject factor of group (BPP vs controls) and the within-subject factor of time. There were no significant differences in placebo responses for any of the DEQ and BAES subscales $(\mathrm{F}<0.5 ; p \geqslant 0.5 ; \mathrm{df}=1)$. Placebo scores were then subtracted from alcohol scores across all time points for the DEQ and BAES subscales (to control further for any possible expectation effects), and then compared using repeated-measures ANOVAs with the between-subjects factors of group and order and the within-subject factor of time. Additionally, participants' maximum ratings on those DEQ and BAES subscales that demonstrated significant between-group differences were selected for correlational analyses with the AEQ subscales, QIDS and BIS-11 scores, in order to explore the relationship between reported intoxication and positive alcohol expectancies, impulsivity and state depressive affect. Finally, given the relative heterogeneity of the selected BPP sample, the above analyses were repeated but without those participants meeting full DSM-IV diagnostic criteria for BD II/NOS, in order to ensure that our results were not attributable to the inclusion of these individuals.

\section{RESULTS}

\section{Demographic and Psychometric Characteristics}

Demographic, psychometric, and alcohol variables are presented in Table 1. There were no significant differences in the age, BMI, IQ, EPQ-N, or weekly alcohol intake of our control participants and our BPP participants. The BPP participants scored a mean of $9.5 \pm 0.37$ on the $M D Q$, indicating significant previous experiences of mood elevation. BPP participants reported significantly more depressive symptoms (QIDS; $\mathrm{F}=5.4, p=0.03$ ) and impulsivity characteristics (BIS-11; $\mathrm{F}=5.7, p=0.02$ ) than the control participants. Three BPP participants met DSM-IVTR diagnostic criteria for BD II or BD NOS at the time of screening, however, none were currently hypomanic at the time of testing. 


\section{Physiological Effects}

BrAC levels following alcohol and placebo treatment are shown in Figure 1. Both participant groups showed similar peak BrAC levels (approximately $0.4 \mu \mathrm{g} / 100 \mathrm{ml}$ at $60 \mathrm{~min}$ ), which declined at similar rates. Similarly, alcohol generally increased pulse and blood pressure following administration of alcohol compared with placebo $(\mathrm{F}=8.9, p=0.005)$. However, BPP participants' pulse and blood pressure did not differ from that of the controls $(\mathrm{F}<0.9 ; p>0.35$; $\mathrm{df}=1$; Supplementary Table S1).

Table I Demographic, Clinical and Alcohol Use Characteristics of HCs $(n=20)$ and Participants with the BPP $(n=20)$

\begin{tabular}{lrrrc}
\hline Clinical characteristics & $\begin{array}{c}\text { HCs }(n=20) \\
\text { Mean } \pm \text { SE }\end{array}$ & BPPs $(n=20)$ & F & P-Value \\
& \multicolumn{5}{c}{} & & \\
\hline Age & $19.21 \pm 0.30$ & $19.32 \pm 0.24$ & 0.07 & 0.79 \\
BMI & $22.89 \pm 0.50$ & $22.10 \pm 0.57$ & 1.00 & 0.32 \\
MDQ & - & $9.45 \pm 0.37$ & - & - \\
Raven's matrices (IQ) & $56.21 \pm 0.71$ & $55.84 \pm 0.84$ & 0.11 & 0.74 \\
Neuroticism (EPQ-N) & $2.55 \pm 0.75$ & $4.05 \pm 0.62$ & 2.38 & 0.13 \\
Impulsivity (BIS-II) & $56.84 \pm 1.92$ & $63.58 \pm 2.18$ & 5.38 & 0.03 \\
Depressive affect (QIDS) & $3.00 \pm 0.46$ & $4.89 \pm 0.64$ & 5.70 & 0.02 \\
Weekly alcohol units & $14.85 \pm 1.48$ & $19.07 \pm 2.09$ & 2.76 & 0.11 \\
\hline
\end{tabular}

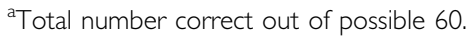

Abbreviations: BIS- I I, Barratt impulsiveness scale I I; BMI, body mass index; BPP, bipolar phenotype participant; EPQ-N, Eysenck personality questionnaire neuroticism subscale; $\mathrm{HC}$, healthy control; $M D Q$, mood disorders questionnaire; QIDS, quick inventory for depressive symptomatology.

Participant groups did not differ in age, BMl, cognitive ability, neuroticism, or weekly alcohol units. Consistent with the phenotype, BPP participants had higher levels of impulsivity (BIS-II) and state depressive affect (QIDS)

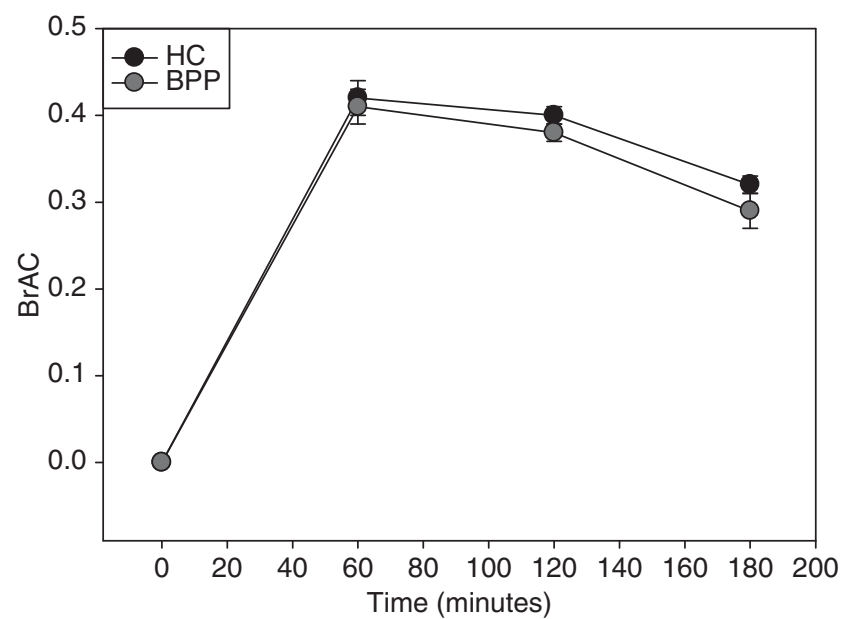

Figure I Mean ( $\pm \mathrm{SE}) \mathrm{BrAC}$ following acute ethanol $(0.8 \mathrm{~g} / \mathrm{kg})$ administration in HCs $(n=20)$ and participants with the BPP $(n=20)$. Both groups obtained similar peak BrAC levels following alcohol administration. Group: NS $(p>0.1)$. BrAC, breath alcohol concentration; BPP, bipolar phenotype participant; HC, healthy control.

\section{Subjective Effects}

No between-group differences in placebo ratings were observed for any of the DEQ or BAES subscales. Following alcohol (over placebo) treatment, BPP participants reported significantly less subjective experiences of intoxication, as assessed by the DEQ subscales 'feel high' $(\mathrm{F}=14.2, p=$ 0.001 ; Figure 2) and 'feel effects' ( $\mathrm{F}=8.1, p=0.008$; Figure 3) in comparison with the control participants. Significant main effects of treatment order were also observed for both 'feel high' and 'feel effects' ( $\mathrm{F}=4.8, p=0.04 ; \mathrm{F}=4.4, p=0.04$, respectively). However, the group by order interactions were not significant for these variables $(\mathrm{F}<1.5 ; p>0.2$; $\mathrm{df}=1$ ). Exclusion of those three participants meeting full DSM-IV criteria for BD II/NOS left our main results unchanged; that is, significant reductions in intoxication reported by the BPP participants in comparison with control participants, as assessed by the DEQ subscales 'feel high' ( $\mathrm{F}=11.4, p=0.002)$ and 'feel effects' $(\mathrm{F}=8.9, p=$ 0.006). Other responses to alcohol (over the placebo) on DEQ subscales including 'like effects' and 'want more', or the BAES stimulation and sedation subscales, were not changed in the BPP participants $(\mathrm{F}<1.7 ; p>0.2 ; \mathrm{df}=1$; Supplementary Figure S1, S2).

Post-hoc correlational analyses revealed neither participants' BIS-11 nor QIDS scores were significantly associated with subjective reports of 'feel effects' or 'feel high' following alcohol compared with placebo treatment in either participant group $(p>0.09 ;-0.6 \leqslant r \leqslant 0.05)$.

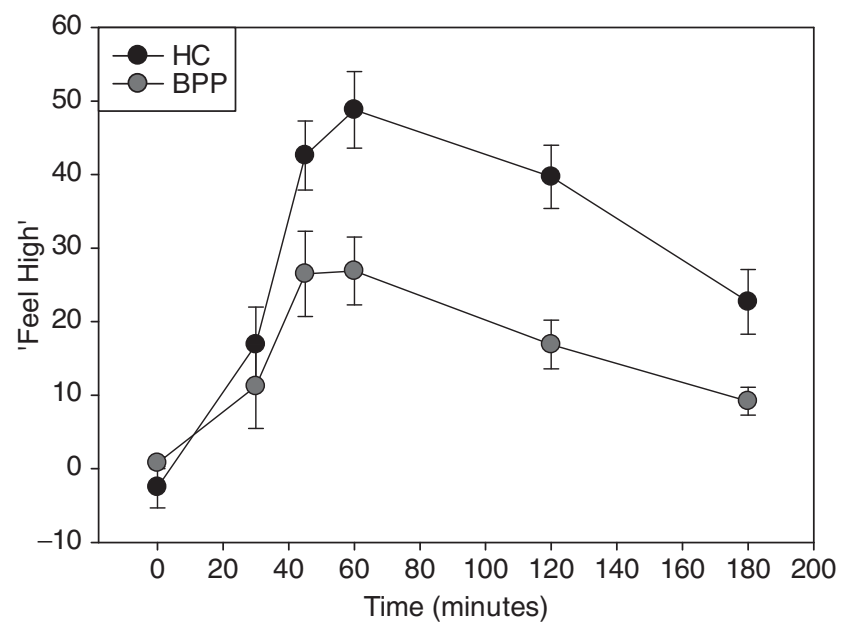

Figure 2 Mean $( \pm \mathrm{SE})$ DEQ 'high' subscale scores across time (alcoholplacebo ratings) following acute ethanol administration $(0.8 \mathrm{~g} / \mathrm{kg})$ in $\mathrm{HCs}$ $(n=20)$ and participants with the BPP $(n=20)$. Beverages (alcohol or placebo) were divided into nine portions and consumed at 3-min intervals, with the last portion consumed at $27 \mathrm{~min}$. BPP participants' ratings of 'feel high' on the DEQ were significantly reduced compared with those of the healthy controls $(F=\mid 4.2 I, p=0.00 \mathrm{I})$, and as a function of time following treatment $(F=4.68, p=0.00$ I). Participants who received alcohol first and placebo second reported higher ratings of 'feel high' than those participants who received the treatments in the reverse order $(F=4.82, p=0.035)$ as a function of time following treatment $(F=3.35, p=0.007)$. However, the three-way interaction between group, order and time was not significant $(F<I ; p>0.1)$. BPP, bipolar phenotype participant; DEQ, drug effects questionnaire; HC, healthy control. 


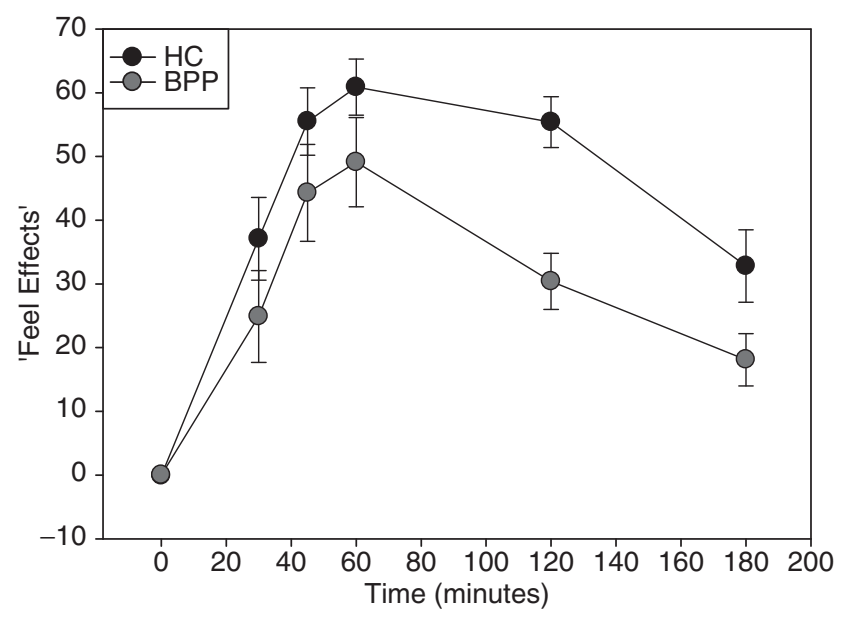

Figure 3 Mean $( \pm \mathrm{SE})$ DEQ 'feel effects' subscale scores across time (alcohol-placebo ratings) following acute ethanol administration $(0.8 \mathrm{~g} / \mathrm{kg})$ in $\mathrm{HCs}(n=20)$ and participants with the BPP $(n=20)$. Beverages (alcohol or placebo) were divided into nine portions and consumed at 3-min intervals, with the last portion consumed at $27 \mathrm{~min}$. BPP participants' ratings of 'feel effects' on the DEQ were significantly lower than those of HCs $(F=8 . I I, p=0.008)$, although the two-way interaction between group and time only approached statistical significance $(F=2.15, p=0.063)$. Participants who received alcohol first and placebo second reported higher ratings of 'feel effects' than those participants who received the treatments in the reverse order $(F=4.4 \mathrm{I}, p=0.044)$. However, neither the two-way interaction between treatment order and time, nor the interaction between group, treatment order and time were significant $(F<1.6$; $p>0.1)$. BPP, bipolar phenotype participant; DEQ, drug effects questionnaire; HC, healthy control.

\section{Alcohol Expectancies}

BPP participants reported significantly more positive alcohol expectancies than the control participants (see Figure 4). This included enhanced expectancies of global positive changes $(\mathrm{F}=9.3, p=0.004)$, relaxation $(\mathrm{F}=8.5, p=0.006)$, and arousal/aggression $(\mathrm{F}=4.8, p=0.03)$, in addition to a trend toward significance for increased sexual enhancement expectancies $(\mathrm{F}=3.5, p=0.07)$. Post-hoc correlational analyses revealed no significant associations between those AEQ subscale scores showing between-group differences and subjective ratings of 'feel effects' and 'feel high' in either participant group $(p>0.09 ;-0.2 \leqslant r \leqslant 0.3)$.

\section{DISCUSSION}

Young people screened in late adolescence and early adulthood report high rates of hypomanic experiences (Chandler et al, 2008; Calabrese et al, 2003) and these experiences are associated with increased rates of mood and anxiety disorders in adulthood including BD (Lewinsohn et al, 2000; Tijssen et al, 2010). Individuals with the BPP are at increased risk for a range of mental health problems. Anxiety and depression are especially common (Chandler et al, 2008). In fact, a number of young people with the BPP already meet DSM-IV-TR criteria for bipolar II or NOS (Rock et al, 2010; Chandler et al, 2009); the symptom profiles and alterations in emotional processing are relatively homogeneous for patients whether or not they meet a syndromal definition of $\mathrm{BD}$.

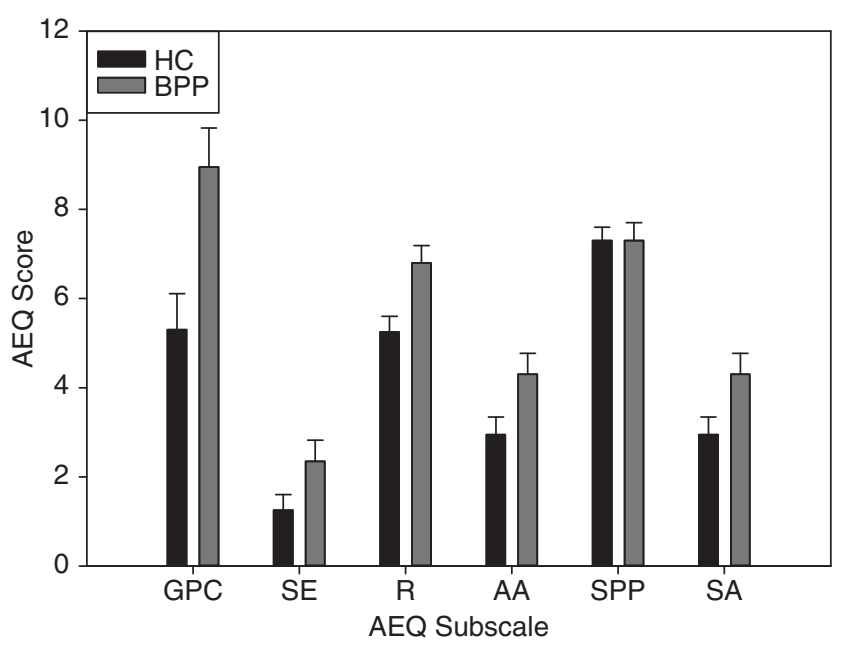

Figure 4 Mean $( \pm$ SE) AEQ subscale scores of HC $(n=20)$ and participants with the BPP $(n=20)$. BPP participants reported significantly more positive alcohol expectancies across multiple domains. GPC: $F=9.36$, $p=0.004 ; S E: F=3.53, p=0.068 ; R: F=8.54, p=0.006 ; A A: F=4.84$, $p=0.034 ;$ SPP: NS $(p>0.1)$; SA: NS $(p>0.1)$. AA, arousal/aggression; $A E Q$, alcohol expectancy questionnaire; GPC, global positive changes; $R$, relaxation; SA, social assertiveness; SE, sexual enhancement; SPP, social and physical pleasure.

In this experiment, young men with the BPP defined as previous experiences of mood elevation reported significantly less subjective intoxication following ethanol administration compared with controls. These differences cannot be attributed to differences in BMI, psychotropic medication, weekly alcohol intake or pharmacokinetic or physiological responses (BrAC and pulse rate). Although significant treatment order effects were observed, these did not interact significantly with participant group or treatment. This means that our between-group differences in subjective responses to alcohol are not attributable to effects in treatment order. Also, the observed differences in subjective responses cannot to be explained by family histories of AUDs. Although this experiment did not explicitly examine the influence of alcohol expectancies, trait impulsivity, and state depressive effect on subjective ratings, the lack of significant associations between subjective responses to alcohol and these variables within either participant group suggests that our findings are not attributable to these factors.

Despite the diminished intoxication ratings, the BPP participants did not differ from the control participants in their self-reported stimulant or sedative responses on either limb of the BrAC curve. These findings are generally consistent with the LLR - but not the differentiatormodel of vulnerability for AUDs (Quinn and Fromme, 2011; Morean and Corbin, 2010), suggesting that individuals with the BPP experienced a generalized decreased awareness of intoxication, which was not biphasic in character. Although the origins of this decreased awareness remain unclear, one possibility is that previous experiences of non-substancerelated mood elevation mean that individuals with BPP are less likely to attribute ethanol-induced mood variation (ie, intoxication) to alcohol.

Individuals with a reduced subjective response to alcohol may have to consume greater quantities of alcohol to obtain 
effects similar to those experienced by their peers, and this has been suggested previously to pre-dispose to AUDs in young people (Schuckit and Smith, 2011). Our findings are consistent with evidence of LLRs to alcohol among young people at increased risk for AUDs (ie, men family history positive for AUDs; Volavka et al, 1996; Schuckit and Smith, 1996; Tolentino et al, 2011). However, our findings extend this possibility to a distinct at-risk group (ie, individuals atrisk for BD) for the first time. LLRs at ages 19 and 20 predict AUDs at 10- and 8-year follow-up (Volavka et al, 1996; Schuckit and Smith, 1996), and are candidate biomarkers for the risk of subsequent alcohol misuse (Tolentino et al, 2011). As such, future studies looking at the longitudinal effects of LLRs among individuals at-risk for BD are warranted. Our findings, nonetheless, suggest that individuals at-risk for BD might be equally at-risk for subsequent AUDs, and further imply possible shared etiological factors between disorders.

Remarkably, the LLRs observed here were clearly present in young people selected exclusively on the basis of their endorsement of hypomanic symptoms in late adolescence. However, despite these diminished experiences of intoxication following alcohol administration under controlled experimental conditions, the same BPP participants also reported significantly elevated alcohol-related expectancies across multiple cognitive domains involving expectations that alcohol will have generalized positive effects. Such increased positive alcohol-related expectancies discriminate between problem $v s$ non-problem-drinkers (Brown et al, 1987) although this has not been demonstrated consistently (Williams and Ricciardelli, 1996). However, given the equivalent levels of current alcohol use in our two participant groups (and the exclusion of participants with a history of alcohol problems), it seems unlikely that the observed differences in alcohol expectancies are attributable to existing drinking patterns. Instead, such expectancies about the effects of alcohol observed among young men with increased rates of hypomanic experiences might promote alcohol consumption, further increasing risk for AUDs. These increased expectancies may relate to cognitive aspects of the bipolar phenotype that may influence behavioral choices that are just starting to be examined including, for example, more intense use of imagery in predicting the impact of future events (Holmes et al, 2011).

\section{Strengths and Limitations}

The robustness of our experiment is under-written by the double-blind, placebo-controlled, randomized, cross-over, within-subjects design, the exclusion of participants taking any psychotropic medication, the relatively young age of the participants (Morean and Corbin, 2010; mean age $\sim 19$ years), and the close similarities in age, IQ, BMI, social milieu, alcohol use, and pharmacokinetic profiles between participant groups. However, notwithstanding these strengths, we note several limitations. First, the status of the BPP remains under examination; it is a marker of risk for-not a simple predictor of - subsequent BD. However, whatever its precise relationship to $\mathrm{BD}$, our findings clearly suggest an increased vulnerability to AUDs among individuals with high rates of hypomanic experiences (ie, individuals with the BPP) but relatively limited family histories of BD (ie, only one BPP participant had a first-degree relative with a history of $\mathrm{BD}$ ). Further studies in individuals more specifically at-risk for of $\mathrm{BD} I$ (such as the siblings of bipolar probands) would extend the generalizability of these findings.

Second, given the familial association between AUDs and BD (Wilens et al, 2007), our exclusion of participants with AUDS, or with family histories of AUDs, may mean that some aspects of the psychopathology associated with the BPP were underestimated. Moreover, findings from some experimental studies of the LLR have been inconsistent (Peterson et al, 1996; Conrod et al, 1997; King et al, 2002, 2011; Newlin and Renton, 2010), with some evidence that it is heightened, rather than reduced, subjective responses to alcohol that are associated with increased risk for AUDs (Thomas et al, 2004). From this perspective, an alternate explanation of our findings is that the BPP individuals included in this study, screened to exclude those individuals with AUDs or family histories of AUDs, are at a lower risk of developing an AUD than individuals having a BPP and co-occurring AUD. From this perspective, the LLR we have observed in our BPP subjects may be a biomarker of that protection. Further experiments involving alcohol administration to healthy control and BPP participants with and without family histories of AUDs are needed to test the secondary hypothesis that subjective responses to alcohol are even lower in those with a positive family history.

Further limitations to this study include the absence of an objective (non-self-report) measure of current alcohol use and the absence of some biological measures of intoxication (eg, body sway), and future studies should consider incorporating these measures. Finally, the absence of female participants means future research is needed to explore any gender-bipolar interactions in subjective responses to alcohol. However, our experiment is consistent with earlier explorations of the LLR model in its inclusion of only male participants (eg, Schuckit and Smith, 1996).

\section{Conclusions}

Although theoretical models conferring vulnerability for cooccurring $\mathrm{BD}$ and AUDs have been proposed, this is the first empirical investigation of subjective responses to alcohol among young people with a phenotype at-risk for $\mathrm{BD}$. The experience of hypomanic symptoms and hypomania is by definition a criterion for bipolar diagnoses and is likely to be relevant to $\mathrm{BD}$ specifically. As all the individuals in this experiment were euthymic, decreased subjective responses to alcohol may be a trait marker for excessive alcohol consumption among individuals with the BPP. The BPP is relatively common (Chandler et al, 2008; Merikangas et al, 2007), so irrespective of the relationship to bipolar diagnoses, the present findings also suggest it may be important in identifying a common and important outcome-alcohol misuse.

\section{ACKNOWLEDGEMENTS}

Financial support for this research was provided by the Medical Research Council (MRC) and the Oxford University Clinical Academic Graduate School (OUCAGS). The authors have no other acknowledgements. 


\section{DISCLOSURE}

Guy M Goodwin holds or has held grants from Bailly Thomas charity, Medical Research Council, NIHR, Servier; has received honoraria from AstraZeneca, BMS, Lundbeck, Sanofi-Aventis, Servier, holds shares in P1vital; has served on advisory boards for AstraZeneca, BMS, Boehringer Ingelheim, Cephalon, Janssen-Cilag, Lilly, Lundbeck, P1Vital, Servier, Shering Plough, Wyeth and acted as an expert witness for Lilly and Servier. Harriet De Wit has received funding from the National Institutes of Health, and for a research study unrelated to this project from Unilever within the last three years. The remaining authors declare no conflict of interest.

\section{REFERENCES}

Brown S, Christiansen B, Goldman M (1987). Alcohol expectancy questionnaire: an instrument for the assessment of adolescent and adult alcohol expectancies. J Stud Alcohol 48: 483-491.

Brown SA (1985). Reinforcement expectancies and alcoholism treatment outcome after a one-year follow-up. J Stud Alcohol 46: 304-308.

Calabrese JR, Hirschfield RM, Reed M, Davies MA, Frye MA, Keck PE et al (2003). Impact of bipolar disorder on a US community sample. J Clin Psychiatry 64: 425-432.

Chandler RA, Wakeley J, Goodwin GM, Rogers RD (2009). Altered risk-aversion and risk-seeking behavior in bipolar disorder. Biol Psychiatry 66: 840-846.

Chandler RA, Wang PW, Ketter TA, Goodwin GM (2008). A new US-UK diagnostic project: mood elevation and depression in first-year undergraduates at Oxford and Stanford universities. Acta Psychiatr Scand 118: 81-85.

Chengappa KR, Levine J, Gershon S, Kupfer DJ (2000). Lifetime prevalence of substance or alcohol abuse and dependence among subjects with bipolar I and II disorders in a voluntary registry. Bipolar Disord 2: 191-195.

Christiansen BA, Smith GT, Roehling PV, Goldman MS (1989). Using alcohol expectancies to predict adolescent drinking behavior after one year. J Consult Clin Psychol 57: 93-99.

Conrod PJ, Peterson JB, Pihl RO, Mankowski S (1997). Biphasic effects of alcohol on heart rate are influenced by alcoholic family history and rate of alcohol ingestion. Alcohol Clin Exp Res 21: 140-149.

Eysenck SBG, Eysenck HJ, Barrett P (1985). A revised version of the psychoticism scale. Pers Individ Dif 6: 21-29.

Frye MA, Altshuler LL, McElroy SL, Suppes T, Keck PE, Denicoff K et al (2003). Gender differences in prevalence, risk, and clinical correlates of alcoholism comorbidity in bipolar disorder. $\mathrm{Am} \mathrm{J}$ Psychiatry 160: 883-889.

Goldman MS, Greenbaum PE, Darkes J (1997). A confirmatory test of hierarchical expectancy structure and predictive power: discriminant validation of the Alcohol Expectancy Questionnaire. Psychol Assess 9: 145-157.

Hirschfeld RMA, Holzer C, Calabrese JR, Weissman M, Reed M, Davies $M$ et al (2003). Validity of the mood disorder questionnaire: a general population study. Am J Psychiatry 160: 178-180.

Hirschfeld RMA, Williams JBW, Spitzer RL, Calabrese JR, Flynn L, Keck PE et al (2000). Development and validation of a screening instrument for bipolar spectrum disorder: the mood disorder questionnaire. Am J Psychiatry 157: 1873-1875.

Holmes EA, Deeprose C, Fairburn CG, Wallace-Hadrill SMA, Bonsall MB, Geddes JR et al. (2011). Mood stability versus mood instability in bipolar disorder: a possible role for emotional mental imagery. Behav Res Ther 49: 707-713.

Kessler RC, Crum RM, Warner LA, Nelson CB, Schulenberg J, Anthony JC (1997). Lifetime co-occurence of DSM-III-R alcohol abuse and dependence with other psychiatric disorders in the National Comorbidity Survey. Arch Gen Psychiatry 54: 313-321.

King AC, de Wit H, McNamara PJ, Cao D (2011). Rewarding, stimulant, and sedative alcohol responses and relationship to future binge drinking. Arch Gen Psychiatry 68: 389-399.

King A, Houle T, de Wit H, Holdstock L, Schuster A (2002). Biphasic alcohol response differs in heavy versus light drinkers. Alcohol Clin Exp Res 26: 827-835.

Kirk J, de Wit $\mathrm{H}$ (2000). Individual differences in the priming effect of ethanol in social drinkers. J Stud Alcohol 61: 64-71.

Le Strat Y, Gorwood P (2008). Does lower response to alcohol explain the high co-morbidity between bipolar disorder and alcohol dependence? Med Hypotheses 71: 81-84.

Lewinsohn PM, Klein DN, Seeley JR (2000). Bipolar disorder during adolescence and young adulthood in a community sample. Bipolar Disord 2: 281-293.

Martin CS, Earleywine M, Musty RE, Perrine MW, Swift RM (1993). Development and validation of the biphasic alcohol effects scale. Alcohol Clin Exp Res 17: 140-146.

Mehrabian A, Russell J (1978). A questionnaire measure of habitual alcohol use. Psychol Rep 43: 803-806.

Merikangas K, Akiskal H, Angst J, Greenberg P, Hirschfeld R, Petukhova $\mathrm{M}$ et al. (2007). Lifetime and 12-month prevalence of bipolar spectrum disorder in the national comorbidity survey replication. Arch Gen Psychiatry 64: 543-552.

Morean M, Corbin W (2010). Subjective response to alcohol: a critical review of the literature. Alcohol Clin Exp Res 34: 385-395.

Newlin DB, Renton RM (2010). High risk groups often have higher levels of alcohol response than low risk: the other side of the coin. Alcohol Clin Exp Res 34: 199-202.

Newlin D, Thompson J (1990). Alcohol challenge with sons of alcoholics: a critical review and analysis. Psychol Bull 108: 383-402.

Patton J, Stanford MS, Barratt ES (1995). Factor structure of the Barratt impulsiveness scale. J Clin Psychology 51: 768-774.

Peterson JB, Pihl RO, Gianoulakis C, Conrod P, Finn PR, Stewart $\mathrm{SH}$ et al (1996). Ethanol-induced change in cardiac and endogenous opiate function and risk for alcoholism. Alcohol Clin Exp Res 20: 1542-1552.

Quinn PD, Fromme K (2011). Subjective response to alcohol challenge: a quantitative review. Alcohol Clin Exp Res 35: 1759-1770.

Raven J, Court J, Raven J. Raven's Progressive Matrices. Psychologists Press: Oxford; (1998).

Ray LA, Mackillop J, Monti PM (2010). Subjective responses to alcohol consumption as endophenotypes: advancing behavioral genetics in etiological and treatment models of alcoholism. Substance Use Misuse 45: 1742-1765.

Regier D, Farmer M, Rae D, Locke B, Keith S, Judd L et al. (1990). Comorbidity of mental disorders with alcohol and other drug abuse. JAMA 264: 2511-2518.

Rock PL, Goodwin GM, Harmer CJ (2010). The common adolescent bipolar phenotype shows positive biases in emotional processing. Bipolar Disord 12: 606-615.

Rueger S, McNamara P, King A (2009). Expanding the utility of the biphasic alcohol effects scale (BAES) and initial psychometric support for the Brief-BAES (B-BAES). Alcohol Clin Exp Res 33: 916-924.

Rush AJ, Trivedi MH, Ibrahim HM, Carmody TJ, Arnow B, Klein $\mathrm{DN}$ et al (2003). The 16-item quick inventory of depressive symptomatology (QIDS), clinician rating (QIDS-C), and selfreport (QIDS-SR): a psychometric evaluation in patients with chronic major depression. Biol Psychiatry 54: 573-583.

Schuckit MA, Smith TL (1996). An 8-year follow-up of 450 sons of alcoholic and control subjects. Arch Gen Psychiatry 53: 202-210.

Schuckit MA, Smith TL (2011). Onset and course of alcoholism over 25 years in middle class men. Drug Alcohol Dependence 113: 21-28.

Sheehan D, Lecrubier Y, Sheehan K, Amorim P, Janavs J, Weiller E et al (1998). The Mini-International Neuropsychiatric Interview 
(M.I.N.I.): the development and validation of a structured diagnostic psychiatric interview for DSM-IV and ICD-10. J Clin Psychiatry 59(Suppl 20): 22-33.

Strakowski SM, Keck PE, McElroy SL, West SA, Sax KW, Hawkins JM et al (1998). Twelve-month outcome after a first hospitalization for affective psychosis. Arch Gen Psychiatry 55: 49-55.

Thomas S, Drobes D, Voronin K, Anton RF (2004). Following alcohol consumption, nontreatment-seeking alcoholics report greater stimulation but not similar sedation compared with social drinkers. J Stud Alcohol 65: 330-335.

Tijssen MJA, van Os J, Wittchen HU, Lieb R, Beesdo K, Mengelers $\mathrm{R}$ et al. (2010). Prediction of transition from common adolescent bipolar experiences to bipolar disorder: 10-year study. $\mathrm{Br} J$ Psychiatry 196: 102-108.

Tohen M, Waternaux CM, Tsuang MT, Hunt AT (1990). Four-year follow-up of twenty-four first-episode manic patients. $J$ Affect Disord 19: 79-86.

Tolentino NJ, Wierenga CE, Hall S, Tapert SF, Paulus MP, Liu TT et al (2011). Alcohol effects on cerebral blood flow in subjects with low and high responses to alcohol. Alcohol Clin Exp Res 35: 1034-1040.
Trim R, Schuckit MA, Smith TL (2009). The relationships of the level of response to alcohol and additional characteristics to alcohol use disorders across adulthood: a discrete-time survival analysis. Alcohol Clin Exp Res 33: 1562-1570.

Volavka J, Czobor P, Goodwin DW, Gabrielli WF, Penick EC, Mednick SA et al (1996). The electroencephalogram after alcohol administration in high-risk men and the development of alcohol use disorders 10 years later: preliminary findings. Arch Gen Psychiatry 53: 258-263.

White TL, Lott DC, de Wit H (2005). Personality and the subjective effects of acute amphetamine in healthy volunteers. Neuropsychopharmacology 31: 1064-1074.

Wilens TE, Biederman J, Adamson J, Monuteaux M, Henin A, Sgambati S et al (2007). Association of bipolar and substance use disorders in parents of adolescents with bipolar disorder. Biol Psychiatry 62: 129-134.

Williams RJ, Ricciardelli LA (1996). Expectancies relate to symptoms of alcohol dependence in young adults. Addiction 91: 1031-1039.

World Health Organization The Global Burden of Disease: 2004 Update. World Health Organization: Switzerland; (2008).

Supplementary Information accompanies the paper on the Neuropsychopharmacology website (http://www.nature.com/npp) 